\title{
ON THE SOLVABILITY OF A CLASS OF SINGULAR PARABOLIC EQUATIONS WITH NONLOCAL BOUNDARY CONDITIONS IN NONCLASSICAL FUNCTION SPACES
}

\author{
ABDELFATAH BOUZIANI
}

Received 11 January 2000

\begin{abstract}
The aim of this paper is to prove the existence, uniqueness, and continuous dependence upon the data of a generalized solution for certain singular parabolic equations with initial and nonlocal boundary conditions. The proof is based on an a priori estimate established in nonclassical function spaces, and on the density of the range of the operator corresponding to the abstract formulation of the considered problem.
\end{abstract}

2000 Mathematics Subject Classification: 35K20, 35B30, 35D05, 46E40, 46 E99.

1. Introduction. This paper is devoted to the solvability of a certain singular parabolic problem with a nonlocal boundary condition. It can be a part in the contribution of the development of the a priori estimates method for solving such problems. The questions related to these problems are so miscellaneous that the elaboration of a general theory is still premature. Therefore, the investigation of these problems requires at every time a separate study.

This work can be considered as a continuation of the results of Yurchuk [12], Benuar and Yurchuk [1], Bouziani [2, 3, 5, 4, 6], Bouziani and Benouar [7, 8], and Mesloub and Bouziani [9], in so far as, on the one hand, the studied equation is parabolic and, on the other hand, the boundary condition is of integral type.

The remainder of the paper is divided into four sections. In Section 2, we give the statement of the problem. Then in Section 3, we first introduce the appropriate function spaces needed in our investigation, the abstract formulation of the problem and the sense of the generalized solution are presented in Section 3.2, and some properties of special smoothing operators are considered in Section 3.3. The uniqueness and the continuous dependence upon the data of a solution are established in Section 4 . In Section 5, the existence of the generalized solution is proved.

2. Statement of the problem. In the rectangle $Q=(0, b) \times(0, T)$, we consider the singular parabolic equation

$$
\mathscr{L} z=\frac{\partial z}{\partial t}-\frac{a(t)}{x} \frac{\partial}{\partial x}\left(x \frac{\partial z}{\partial x}\right)=f(x, t)
$$

where $b$ and $T$ are fixed but arbitrary positive numbers, and $a(t)$ is a known function satisfying the following assumption. 
Assumption 2.1. For $t \in[0, T]$, we assume that

$$
c_{0} \leq a(t) \leq c_{1}, \quad a^{\prime}(t) \leq c_{2} .
$$

In Assumption 2.1, and throughout, we suppose that $c_{i}$ (where $i=0, \ldots, 4$ ) are positive constants.

We pose the following problem for (2.1): given the data $f, \Phi, \mu$, and $M$, find a function $z=z(x, t)$ subject to the initial condition

$$
\ell z=z(x, 0)=\Phi(x), \quad \text { for } 0 \leq x \leq b,
$$

the Dirichlet condition

$$
z(b, t)=\mu(t), \quad \text { for } 0 \leq t \leq T, \quad \Phi(b)=\mu(0),
$$

and the weighted integral condition

$$
\int_{0}^{b} x^{2} z(x, t) d x=M(t) \quad \text { for } 0 \leq x \leq b, \quad \int_{0}^{b} x^{2} \Phi(x) d x=M(0) .
$$

We transform problem (2.1), (2.3), (2.4), and (2.5) with inhomogeneous boundary conditions into a problem with homogeneous boundary conditions. For this, we put $z(x, t)=u(x, t)+\zeta(x, t)$, where

$$
\zeta(x, t)=\frac{x}{b} \mu(t)+\frac{12}{b^{4}}(b-x)\left(M(t)-\frac{b^{3}}{4} \mu(t)\right) .
$$

Then, problem (2.1), (2.3), (2.4), and (2.5) can be transformed as follows: find a function $u=u(x, t)$ satisfying

$$
\begin{gathered}
\mathscr{L} u=\frac{\partial u}{\partial t}-\frac{a(t)}{x} \frac{\partial}{\partial x}\left(x \frac{\partial u}{\partial x}\right)=f(x, t)-\mathscr{L} \zeta=f(x, t), \\
\ell u=u(x, 0)=\Phi(x)-\ell \zeta=\varphi(x), \\
u(b, t)=0 \\
\int_{0}^{b} x^{2} u(x, t) d x=0 .
\end{gathered}
$$

\section{Preliminaries}

3.1. Function spaces. We first introduce appropriate function spaces. We denote by $C_{0}(0, b)$ the vector space of continuous functions with compact support in $(0, b)$. Since such functions are Lebesgue integrable with respect to $d x$, we can define on $C_{0}(0, b)$ the bilinear form $((\cdot, \cdot))_{x}$ given by

$$
((u, w))_{x}=\int_{0}^{b} \mathfrak{I}_{x}^{*}(\xi u) \cdot \mathfrak{I}_{x}^{*}(\xi w) d x
$$

where $\mathfrak{J}_{x}^{*} \mathfrak{g}=\int_{x}^{b} \mathfrak{g}(\xi, t) d \xi$. We recall that $((\cdot, \cdot))_{x}$ is a scalar product on $C_{0}(0, b)$ for which $C_{0}(0, b)$ is not complete. Thus we are led to introduce its completion. 
DEFINITION 3.1. We denote by $B_{2, x}^{1, *}(0, b)$ a completion of $C_{0}(0, b)$ for the scalar product defined by (3.1), called the space of square integrable weighted primitive functions on $(0, b)$ (or the weighted Bouziani space).

REMARK 3.2. If $x=1$, then the space $B_{2, x}^{1, *}(0, b)$ is identified with the space $B_{2}^{1, *}(0, b)$, first introduced in $[2,5]$.

Definition 3.3. We denote by $B_{2, \rho}^{1}(0, b)$ a completion of $C_{0}(0, b)$ for the scalar product defined by

$$
((u, w))_{\rho}=\int_{0}^{T} \mathfrak{I}_{t}(\rho(\tau) u) \cdot \mathfrak{I}_{t}(\rho(\tau) w) d t
$$

where $\mathfrak{I}_{t} \mathfrak{g}=\int_{0}^{t} \mathfrak{g}(x, \tau) d \tau$ and $\rho(t)=e^{c t}$.

Now, we generalize Definitions 3.1 and 3.3 of weighted Bouziani spaces $B_{2, x}^{1, *}(0, b)$ and $B_{2, \rho}^{1}(0, T)$.

DeFinition 3.4. Let $(0, b)$ (resp., $(0, T)$ ) be an open interval in $\mathbb{R}$, let $\sigma(x)$ (resp., $\rho(t)$ ) be a continuous function from $(0, b)$ to $\mathbb{R}_{*}^{+}$(resp., from $(0, T)$ to $\mathbb{R}_{*}^{+}$), let $m$ be a non-negative integer and let $1 \leq p \leq \infty$. Then we define $B_{p, \sigma}^{m, *}(0, b)\left(\operatorname{resp} ., B_{p, \rho}^{m}(0, T)\right)$ to be the completion of the space $C_{0}(0, b)$ (resp., $C_{0}(0, T)$ ) for the norm

$$
\|u\|_{B_{p, \sigma}^{m, *}(0, b)}=\left\{\int_{0}^{b}\left(\mathfrak{J}_{x}^{*^{m}}(\sigma u)\right)^{p} d x\right\}^{1 / p},
$$

respectively,

$$
\|u\|_{B_{p, \rho}^{m}(0, T)}=\left\{\int_{0}^{T}\left(\mathfrak{J}_{t}^{m}(\rho u)\right)^{p} d t\right\}^{1 / p},
$$

and for $p=2$, we define a scalar product by

$$
(u, w)_{B_{2, \sigma}^{m, *}(0, b)}=\left(\mathfrak{J}_{x}^{*^{m}}(\sigma u), \mathfrak{J}_{x}^{*^{m}}(\sigma w)\right)_{L^{2}(0, b)},
$$

respectively,

$$
(u, w)_{B_{2, \rho}^{m}(0, T)}=\left(\mathfrak{J}_{t}^{m}(\rho u), \mathfrak{J}_{t}^{m}(\rho w)\right)_{L^{2}(0, T)} .
$$

REMARK 3.5. The spaces $B_{2, \sigma}^{0, *}(0, b)$ and $B_{2, \rho}^{0}(0, T)$ coincide (with equality of norm of graphs) with the spaces $L_{x}^{2}(0, b)$ and $L_{\rho}^{2}(0, T)$, respectively; that is, by the norms of functions $u$ from $L_{x}^{2}(0, b)$ and $L_{\rho}^{2}(0, T)$ we understand the nonnegative numbers: $\|u\|_{L_{X}^{2}(0, b)}=\left\{\int_{0}^{b}(x u)^{2} d x\right\}^{1 / 2}$ and $\|u\|_{L_{\rho}^{2}(0, T)}=\left\{\int_{0}^{T}(\rho(t) u)^{2} d t\right\}^{1 / 2}$, respectively.

In this paper, we also use other weighted spaces such as $L_{\sigma}^{2}(0, b), L_{s}^{2}(0, b)$, and $L_{r}^{2}(0, T)$, where $\sigma(x)=x^{2}, s(x)=\sqrt{x}$, and $r(t)=\sqrt{\rho(t)}=e^{c t / 2}$, which are Hilbert spaces of (classes of) weighted square integrable functions with finite norms:

$$
\begin{aligned}
\|u\|_{L_{\sigma}^{2}(0, b)} & =\left\{\int_{0}^{b}(\sigma u)^{2} d x\right\}^{1 / 2}, \\
\|u\|_{L_{S}^{2}(0, b)} & =\left\{\int_{0}^{b}(s u)^{2} d x\right\}^{1 / 2}, \\
\|u\|_{L_{r}^{2}(0, T)} & =\left\{\int_{0}^{T}(r u)^{2} d t\right\}^{1 / 2} .
\end{aligned}
$$


Let $H_{s}^{1}(0, b)=\left\{u / u \in L_{s}^{2}(0, b), \partial u / \partial x \in L_{s}^{2}(0, b), \int_{0}^{b} x^{2} u(x, t) d x=0\right\}$, which is the Hilbert space for the norm

$$
\|u\|_{H_{S}^{1}(0, b)}=\left\{\|u\|_{L_{S}^{2}(0, b)}^{2}+\left\|\frac{\partial u}{\partial x}\right\|_{L_{S}^{2}(0, b)}^{2}\right\}^{1 / 2}
$$

Let $H$ be a Hilbert space with a norm $\|\cdot\|_{H}$. We denote by $L^{2}(0, T ; H)\left(\operatorname{resp} ., L_{r}^{2}(0, T ; H)\right)$ the set of all measurable abstract functions $u(\cdot, t)$ from $(0, T)$ into $H$ such that

$$
\|u\|_{L^{2}(0, T ; H)}=\left\{\int_{0}^{T}\|u(\cdot, t)\|_{H}^{2} d t\right\}^{1 / 2}<\infty
$$

respectively,

$$
\|u\|_{L_{r}^{2}(0, T ; H)}=\left\{\int_{0}^{T}\left(e^{c t / 2}\|u(\cdot, t)\|_{H}\right)^{2} d t\right\}^{1 / 2}<\infty .
$$

Let $C(0, T ; H)$ be the set of all continuous functions $u(\cdot, t):(0, T) \rightarrow H$ with

$$
\|u\|_{C(0, T ; H)}=\sup _{0 \leq \tau \leq T}\|u(\cdot, \tau)\|_{H}<\infty .
$$

We write $B_{2, \rho}^{1}(0, T ; H)$ for the space of functions from $(0, T)$ into $H$ which are weighted Bouziani space for the measure $d t$. It is a Hilbert space for the norm

$$
\|u\|_{B_{2, \rho}^{1}(0, T ; H)}=\left\{\int_{0}^{T}\left(\mathfrak{J}_{t}\left(e^{c \tau}\|u(\cdot, \tau)\|_{H}\right)\right)^{2} d t\right\}^{1 / 2}
$$

The following inequalities are well known and are frequently used in this paper. We list them here for convenience.

LEMMA 3.6. For $x \in(0, b)$, the following inequalities hold:

$$
\begin{aligned}
\|u\|_{L_{x}^{2}(0, b)}^{2} & \leq b\|u\|_{L_{S}^{2}(0, b)}^{2}, \\
\|u\|_{B_{2}^{1, *}(0, b)}^{2} & \leq 4\|u\|_{L_{x}^{2}(0, b)}^{2}, \\
\|u\|_{B_{2, x}^{1, *}(0, b)}^{2} & \leq \frac{b^{2}}{2}\|u\|_{L_{x}^{2}(0, b)}^{2} .
\end{aligned}
$$

We are now in a position to give the abstract formulation corresponding to the problem (2.7), (2.8), and (2.9).

3.2. Abstract formulation. We consider problem (2.7), (2.8), and (2.9) as the solution of the abstract equation

$$
L u=(f, \varphi),
$$


where $L$ is the operator which maps $u(x, t)$ to the pair of elements $\mathscr{L} u$ and $\ell u$, so that

$$
L u=(\mathscr{L} u, \ell u) .
$$

We consider $L$ as an unbounded operator with domain $D(L)$ consisting of all functions $u$ belonging to $L^{2}\left(0, T ; B_{2, x}^{1, *}(0, b)\right)$ for which $\partial u / \partial t,(1 / x)(\partial u / \partial x), \partial^{2} u / \partial x^{2} \in$ $L^{2}\left(0, T ; B_{2, x}^{1, *}(0, b)\right)$ and satisfying conditions (2.9). We complete $D(L)$ in the norm

$$
\|u\|_{B}=\left\{\left\|\frac{\partial u}{\partial t}\right\|_{L^{2}\left(0, T ; B_{2, x}^{1, *}(0, b)\right)}^{2}+\|u\|_{C\left(0, T ; H_{S}^{1}(0, b)\right)}^{2}\right\}^{1 / 2} ;
$$

this yields a Banach space $B$. The elements of $B$ are continuous functions on $[0, T]$ with values in $H_{s}^{1}(0, b)$. Hence on $B$, the following mapping is defined and continuous:

$$
\ell: B \ni u \longrightarrow \ell u=u(x, 0) \in H_{s}^{1}(0, b) .
$$

We write $F$ for the Hilbert space $L^{2}\left(0, T ; L_{s}^{2}(0, b)\right) \times H_{s}^{1}(0, b)$ consisting of all elements $(f, \varphi)$ for which the norm

$$
\|(f, \varphi)\|_{F}=\left\{\|f\|_{L^{2}\left(0, T ; L_{S}^{2}(0, b)\right)}^{2}+\|\varphi\|_{H_{S}^{1}(0, b)}^{2}\right\}^{1 / 2}
$$

is finite. We consider the operator $L$ with the above domain as a mapping from $B$ into $F$.

Now, we can introduce the concept of a generalized solution of problem (2.7), (2.8), and (2.9). Let $\bar{L}$ be the closure of the operator $L$.

DEFINITION 3.7. A solution of the operator equation

$$
\bar{L} u=(f, \varphi), \quad(f, \varphi) \in F,
$$

is called a generalized solution of problem (2.7), (2.8), and (2.9).

To prove the solvability of problem (2.7), (2.8), and (2.9) in the sense of Definition 3.7, we establish the a priori estimate

$$
\|u\|_{B} \leq c\|L u\|_{F}, \quad u \in D(L) .
$$

It follows from (3.21) that there is a bounded inverse $L^{-1}$ on the range $R(L)$ of $L$. However, since we have no information concerning $R(L)$ except that $R(L) \subset F$, we must extend $L$, so that an a priori estimate like (3.21) holds for the extension. For this, we prove that $L$ admits a closure. Thus we extend (3.21) to $u \in D(\bar{L})$ by passing to the limit. It follows that the closure procedure for $L$ reduces to the closure of the range $R(L)$ in $F$, so that $\overline{R(L)}=R(\bar{L})$, and a bounded inverse $\bar{L}^{-1}$ exists on $R(\bar{L})$, so the uniqueness of a generalized solution. For existence, it remains to prove that $R(L)$ does not have an orthogonal complement in $F$.

3.3. Smoothing operators. We consider the operators defined by the relations

$$
\begin{gathered}
\left(\rho_{\varepsilon}^{-1}\right) v=\frac{1}{\varepsilon} \mathfrak{J}_{t}\left(e^{(1 / \varepsilon)(\tau-t)} v\right), \quad \varepsilon>0, \\
\left(\rho_{\varepsilon}^{-1}\right)^{*} v=-\frac{1}{\varepsilon} \mathfrak{J}_{t}^{*}\left(e^{(1 / \varepsilon)(t-\tau)} v\right), \quad \varepsilon>0,
\end{gathered}
$$


where $\mathfrak{J}_{t}^{*} \mathfrak{g}=\int_{t}^{T} \mathfrak{g}(x, \tau) d \tau$. These operators, first proposed by Yurchuk in abstract form in [11], are used as smoothing operators with respect to $t$ [12]. They furnish the solutions of the problems

$$
\begin{gathered}
\varepsilon \frac{\partial\left(\rho_{\varepsilon}^{-1}\right) v}{\partial t}+\left(\rho_{\varepsilon}^{-1}\right) v=v, \quad\left(\rho_{\varepsilon}^{-1}\right) v(x, 0)=0, \\
-\varepsilon \frac{\partial\left(\rho_{\varepsilon}^{-1}\right)^{*} v}{\partial t}+\left(\rho_{\varepsilon}^{-1}\right)^{*} v=v, \quad\left(\rho_{\varepsilon}^{-1}\right)^{*} v(x, T)=0,
\end{gathered}
$$

respectively. These operators have, for all $v \in L^{2}\left(0, T, L^{2}(0, b)\right)$, the following properties:

(P1) the functions $\left(\rho_{\varepsilon}^{-1}\right) v$ and $\left(\rho_{\varepsilon}^{-1}\right)^{*} v \in H^{1}(0, T)$, with $\left(\rho_{\varepsilon}^{-1}\right) v(x, 0)=0$, and $\left(\rho_{\varepsilon}^{-1}\right)^{*} v(x, T)=0$

(P2) the operators $\left(\rho_{\varepsilon}^{-1}\right)^{*}$ are conjugate to $\left(\rho_{\varepsilon}^{-1}\right)$, that is,

$$
\int_{Q}\left(\rho_{\varepsilon}^{-1}\right) v \cdot \omega d x d t=\int_{Q} v \cdot\left(\rho_{\varepsilon}^{-1}\right)^{*} \omega d x d t, \quad \forall \omega \in L^{2}(0, T) ;
$$

(P3) $\left(\rho_{\varepsilon}^{-1}\right)(\partial v / \partial T)=(\partial / \partial t)\left(\rho_{\varepsilon}^{-1}\right) v+(1 / \varepsilon) e^{-t / \varepsilon} \cdot v(x, 0)$;

(P4) $\int_{0}^{T}\left\|\left(\rho_{\varepsilon}^{-1}\right) v\right\|_{L^{2}(0, b)} d t \leq \int_{0}^{T}\|v\|_{L^{2}(0, b)} d t$ and $\int_{0}^{T}\left\|\left(\rho_{\varepsilon}^{-1}\right) v-v\right\|_{L^{2}(0, b)} d t \rightarrow 0$, when $\varepsilon \rightarrow 0$;

(P5) $\int_{0}^{T}\left\|\left(\rho_{\varepsilon}^{-1}\right)^{*} v\right\|_{L^{2}(0, b)} d t \leq \int_{0}^{T}\|v\|_{L^{2}(0, b)} d t$ and $\int_{0}^{T}\left\|\left(\rho_{\varepsilon}^{-1}\right)^{*} v-v\right\|_{L^{2}(0, b)} d t \rightarrow 0$, when $\varepsilon \rightarrow 0$

(P6) if $A(t) v=a(t)(\partial / \partial x)(x(\partial v / \partial x))$ then

$$
A(t)\left(\rho_{\varepsilon}^{-1}\right) v=\left(\rho_{\varepsilon}^{-1}\right) A(\tau) v+\varepsilon\left(\rho_{\varepsilon}^{-1}\right) A^{\prime}(\tau)\left(\rho_{\varepsilon}^{-1}\right) v
$$

where $A^{\prime}(t) v=a^{\prime}(t)(\partial / \partial x)(x(\partial u / \partial x))$.

For the proof of these properties, see, for instance, [4].

4. Uniqueness and continuous dependence. In this section, we first establish an a priori estimate. The uniqueness and the continuous dependence of the solution upon the data then are direct corollary of it.

THEOREM 4.1. Under Assumption 2.1, the solution of problem (2.7), (2.8), and (2.9) satisfies the following a priori estimate:

$$
\|u\|_{B} \leq c\|L u\|_{F},
$$

where $c$ is a positive constant independent of $u$.

Proof. We consider the scalar product in $L^{2}\left(0, \tau ; B_{2, x}^{1, *}(0, b) \cap L_{s}^{2}(0, b)\right)$, with $0 \leq$ $\tau \leq T$, of (2.7) and $\partial u / \partial t$, yields

$$
\begin{gathered}
\int_{0}^{\tau}\left\|\frac{\partial u(\cdot, t)}{\partial t}\right\|_{B_{2, x}^{1, *}(0, b)}^{2} d t-\int_{0}^{\tau}\left(\frac{a(t)}{x} \frac{\partial}{\partial x}\left(x \frac{\partial u(\cdot, t)}{\partial x}\right), \frac{\partial u(\cdot, t)}{\partial t}\right)_{B_{2, x}^{1, *}(0, b)} d t \\
\quad+\int_{0}^{\tau}\left\|\frac{\partial u(\cdot, t)}{\partial t}\right\|_{L_{S}^{2}(0, b)}^{2} d t-\int_{0}^{\tau}\left(\frac{a(t)}{x} \frac{\partial}{\partial x}\left(x \frac{\partial u(\cdot, t)}{\partial x}\right), \frac{\partial u(\cdot, t)}{\partial t}\right)_{L_{S}^{2}(0, b)} d t \\
=\int_{0}^{\tau}\left(f(\cdot, t), \frac{\partial u(\cdot, t)}{\partial t}\right)_{B_{2, x}^{1, *}(0, b)} d t+\int_{0}^{\tau}\left(f(\cdot, t), \frac{\partial u(\cdot, t)}{\partial t}\right)_{L_{S}^{2}(0, b)} d t
\end{gathered}
$$


The standard integration by parts of the second and last terms on the left-hand side of (4.2) leads to

$$
\begin{aligned}
-\int_{0}^{\tau}\left(\frac{a(t)}{x} \frac{\partial}{\partial x}\left(x \frac{\partial u(\cdot, t)}{\partial x}\right), \frac{\partial u(\cdot, t)}{\partial t}\right)_{B_{2, x}^{1, *}(0, b)} d t & =\int_{0}^{\tau} \int_{0}^{b} a(t) x \frac{\partial u}{\partial x} \mathfrak{J}_{x}^{*}\left(\xi \frac{\partial u}{\partial t}\right) d x d t \\
-\int_{0}^{\tau}\left(\frac{a(t)}{x} \frac{\partial}{\partial x}\left(x \frac{\partial u(\cdot, t)}{\partial x}\right), \frac{\partial u(\cdot, t)}{\partial t}\right)_{L_{S}^{2}(0, b)} d t & =\frac{1}{2} \int_{0}^{b} a(\tau) x\left(\frac{\partial u(x, \tau)}{\partial x}\right)^{2} d x \\
& -\frac{1}{2} \int_{0}^{b} a(0) x\left(\frac{d \varphi}{d x}\right)^{2} d x \\
& -\frac{1}{2} \int_{0}^{\tau} \int_{0}^{b} a^{\prime}(t) x\left(\frac{\partial u}{\partial x}\right)^{2} d x d t
\end{aligned}
$$

Substituting (4.3) into (4.2), we get

$$
\begin{aligned}
\int_{0}^{\tau} \| & \frac{\partial u(\cdot, t)}{\partial t}\left\|_{B_{2, x}^{1, *}(0, b)}^{2} d t+\int_{0}^{\tau}\right\| \frac{\partial u(\cdot, t)}{\partial t} \|_{L_{S}^{2}(0, b)}^{2} d t+\frac{1}{2} \int_{0}^{b} a(\tau) x\left(\frac{\partial u(x, \tau)}{\partial x}\right)^{2} d x \\
= & \int_{0}^{\tau}\left(f, \frac{\partial u}{\partial t}\right)_{B_{2, x}^{1, *}(0, b)} d t+\int_{0}^{\tau}\left(f(\cdot, t), \frac{\partial u(\cdot, t)}{\partial t}\right)_{L_{S}^{2}(0, b)} d t+\frac{1}{2} \int_{0}^{b} a(0) x\left(\frac{d \varphi}{d x}\right)^{2} d x \\
& +\frac{1}{2} \int_{0}^{\tau} \int_{0}^{b} a^{\prime}(t) x\left(\frac{\partial u}{\partial x}\right)^{2} d x d t-\int_{0}^{\tau} \int_{0}^{b} a(t) x \frac{\partial u}{\partial x} \mathfrak{J}_{x}^{*}\left(\xi \frac{\partial u}{\partial t}\right) d x d t .
\end{aligned}
$$

In light of the Cauchy inequality and inequality (3.13), the first two terms and the last term in the right-hand side of (4.4) are then majorized as follows:

$$
\begin{aligned}
& \int_{0}^{T}\left(f(\cdot, t), \frac{\partial u(\cdot, t)}{\partial t}\right)_{B_{2, x}^{1, *}(0, b)} d t \\
& \quad \leq \frac{\varepsilon_{1}}{2} \int_{0}^{T}\|f(\cdot, t)\|_{B_{2, x}^{1, *}(0, b)}^{2} d t+\frac{1}{2 \varepsilon_{1}} \int_{0}^{\tau}\left\|\frac{\partial u(\cdot, t)}{\partial t}\right\|_{B_{2, x}^{1, *}(0, b)}^{2} d t \\
& \int_{0}^{\tau}\left(f(\cdot, t), \frac{\partial u(\cdot, t)}{\partial t}\right)_{L_{S}^{2}(0, b)} d t \\
& \quad \leq \frac{\varepsilon_{2}}{2} \int_{0}^{\tau}\|f(\cdot, t)\|_{L_{S}^{2}(0, b)}^{2} d t+\frac{1}{2 \varepsilon_{2}} \int_{0}^{\tau}\left\|\frac{\partial u(\cdot, t)}{\partial t}\right\|_{L_{S}^{2}(0, b)}^{2} d t, \\
& \quad \int_{0}^{T} \int_{0}^{b} a(t) x \frac{\partial u}{\partial x} \mathfrak{J}_{x}^{*}\left(\xi \frac{\partial u}{\partial t}\right) d x d t \\
& \quad \leq \frac{b \varepsilon_{3}}{2} \int_{0}^{\tau} a^{2}(t)\left\|\frac{\partial u(\cdot, t)}{\partial x}\right\|_{L_{S}^{2}(0, b)}^{2} d t+\frac{1}{2 \varepsilon_{3}} \int_{0}^{T}\left\|\frac{\partial u(\cdot, t)}{\partial t}\right\|_{B_{2, x}^{1, *}(0, b)}^{2} d t .
\end{aligned}
$$


Combining the inequalities (4.5) with (4.4), choosing $\varepsilon_{1}=3 / 2, \varepsilon_{2}=3 / 4$, and $\varepsilon_{3}=3 / 2$, and using Assumption 2.1, we obtain

$$
\begin{aligned}
\frac{1}{3} \int_{0}^{\tau}\left\{\left\|\frac{\partial u(\cdot, t)}{\partial t}\right\|_{B_{2, x}^{1, *}(0, b)}^{2}+\left\|\frac{\partial u(\cdot, t)}{\partial t}\right\|_{L_{S}^{2}(0, b)}^{2}\right\} d t+\frac{c_{0}}{2}\left\|\frac{\partial u(\cdot, \tau)}{\partial x}\right\|_{L_{S}^{2}(0, b)}^{2} \\
\leq \frac{3}{4} \int_{0}^{\tau}\|f(\cdot, t)\|_{B_{2, x}^{1, *}(0, b)}^{2} d t+\frac{3}{8} \int_{0}^{\tau}\|f(\cdot, t)\|_{L_{S}^{2}(0, b)}^{2} d t+\frac{c_{1}}{2}\left\|\frac{d \varphi}{d x}\right\|_{L_{S}^{2}(0, b)}^{2} \\
+\frac{3 b c_{1}^{2}}{4} \int_{0}^{\tau}\left\|\frac{\partial u(\cdot, t)}{\partial x}\right\|_{L_{S}^{2}(0, b)}^{2} d t
\end{aligned}
$$

Observing that

$$
\frac{1}{3}\|u(\cdot, \tau)\|_{L_{S}^{2}(0, b)}^{2} \leq \frac{1}{3}\|\varphi\|_{L_{S}^{2}(0, b)}^{2}+\frac{1}{3} \int_{0}^{\tau}\|u(\cdot, t)\|_{L_{S}^{2}(0, b)}^{2} d t+\frac{1}{3} \int_{0}^{\tau}\left\|\frac{\partial u(\cdot, t)}{\partial t}\right\|_{L_{S}^{2}(0, b)}^{2} d t
$$

it follows by using (3.13) and (3.14) that

$$
\begin{aligned}
& \int_{0}^{\tau}\left\|\frac{\partial u(\cdot, t)}{\partial t}\right\|_{B_{2, x}^{1, *}(0, b)}^{2} d t+\|u(\cdot, \tau)\|_{H_{S}^{1}(0, b)}^{2} \\
& \quad \leq c_{3}\left(\int_{0}^{\tau}\|f(\cdot, t)\|_{L_{S}^{2}(0, b)}^{2} d t+\|\varphi\|_{H_{S}^{1}(0, b)}^{2}\right)+c_{4} \int_{0}^{\tau}\|u(\cdot, t)\|_{H_{S}^{1}(0, b)}^{2} d t
\end{aligned}
$$

where

$$
c_{3}=\frac{\max \left(c_{1} / 2,3\left(1+b^{3}\right) / 8\right)}{\min \left(1 / 3, c_{0} / 2\right)}, \quad c_{4}=\frac{\max \left(1 / 3,3 b c_{1}^{2} / 4\right)}{\min \left(1 / 3, c_{0} / 2\right)} .
$$

We eliminate the last term on the right-hand side of (4.8). To do that we use [3, Lemma 3.1] to obtain

$$
\begin{aligned}
& \int_{0}^{T}\left\|\frac{\partial u(\cdot, t)}{\partial t}\right\|_{B_{2, x}^{1, *}(0, b)}^{2} d t+\|u(\cdot, \tau)\|_{H_{S}^{1}(0, b)}^{2} \\
& \quad \leq c_{3} \exp \left(c_{4} T\right)\left(\int_{0}^{T}-\|f(\cdot, t)\|_{L_{S}^{2}(0, b)}^{2} d t+\|\varphi\|_{H_{S}^{1}(0, b)}^{2}\right) .
\end{aligned}
$$

Since the right-hand side here does not depend on $\tau$; we take the upper bound of the left-hand side on $\tau$ from 0 to $T$; hence (4.1) holds with $c=c_{3}^{1 / 2} \exp \left(c_{4} T / 2\right)$, and this proves Theorem 4.1 .

We show that the operator $L$ admits a closure, that is, the closure of the graph $G(L) \subset B \times F$ of $L$ is a graph $G(\bar{L})=\overline{G(L)}$ of operator $\bar{L}$.

Proposition 4.2. The operator $L: B \rightarrow F$ with domain $D(L)$ has a closure.

Proof. For the proof, the reader should refer to [10]. 
We extend the a priori estimate (4.1) to $u \in D(\bar{L})$ by passing to the limit, that is,

$$
\|u\|_{B} \leq c\|\bar{L} u\|_{F}, \quad \forall u \in D(\bar{L}) .
$$

From (4.11) we conclude the following corollaries.

COROLlary 4.3. Let the conditions of Theorem 4.1 be satisfied. If problem (2.7), (2.8), and (2.9), has a generalized solution, then this solution is unique and depends continuously on $(f, \varphi)$.

COROLLARY 4.4. The range $R(\bar{L})$ of $\bar{L}$ is closed in $F$ and $R(\bar{L})=\overline{R(L)}$, where $R(L)$ is the range of $L$.

5. The existence of the solution. Now we want to prove the solvability of our problem. Our existence theorem reads as follows.

THEOREM 5.1. There exists a function $u \in C\left(0, T ; H_{s}^{1}(0, b)\right)$ with $\partial u / \partial t \in L^{2}(0, T$; $\left.B_{2, x}^{1, *}(0, b)\right)$ which solves problem (2.7), (2.8), and (2.9) in the sense of Definition 3.7, for arbitrary $f \in L^{2}\left(0, T ; L_{s}^{2}(0, b)\right)$ and $\varphi \in H_{s}^{1}(0, b)$.

Proof. Corollary 4.4 shows that, to prove that (2.7), (2.8), and (2.9) has a generalized solution for each $(f, \varphi) \in F$, it is sufficient to show that $R(L)$ is dense in $F$. For this we need the following proposition.

Proposition 5.2. If

$$
\int_{0}^{T}(\mathscr{L} u(\cdot, t), \omega(\cdot, t))_{L_{S}^{2}(0, b)} d t=0
$$

for some function $\omega \in L^{2}\left(0, T ; L_{s}^{2}(0, b)\right)$ and all $u \in D_{0}(L)=\{u / u \in D(L): \ell u=0\}$, then $\omega \equiv 0$ almost everywhere in $Q$.

Proof Of The Proposition. Equation (5.1) may be written in the form

$$
\int_{0}^{T}\left(\frac{\partial u(\cdot, t)}{\partial t}, \omega(\cdot, t)\right)_{L_{S}^{2}(0, b)} d t=\int_{0}^{T}(A(t) u, \omega(\cdot, t))_{L^{2}(0, b)} d t .
$$

Substitute in (5.2) $u$ by the smooth function $\rho_{\varepsilon}^{-1} u$, hence by property (P3) it follows that

$$
\int_{0}^{T}\left(\rho_{\varepsilon}^{-1} \frac{\partial u}{\partial \tau}, \omega(\cdot, t)\right)_{L_{S}^{2}(0, b)} d t=\int_{0}^{T}\left(A(t) \rho_{\varepsilon}^{-1} u, \omega(\cdot, t)\right)_{L^{2}(0, b)} d t .
$$

Applying property (P6) to the right-hand side of (5.3), we get

$$
\int_{0}^{T}\left(\rho_{\varepsilon}^{-1} \frac{\partial u}{\partial \tau}, \omega(\cdot, t)\right)_{L_{S}^{2}(0, b)} d t=\int_{0}^{T}\left(\rho_{\varepsilon}^{-1} A(t) u+\varepsilon \rho_{\varepsilon}^{-1} A^{\prime}(t) \rho_{\varepsilon}^{-1} u, \omega(\cdot, t)\right)_{L^{2}(0, b)} d t .
$$

According to property (P2), it follows that

$$
\int_{0}^{T}\left(\frac{\partial u(\cdot, t)}{\partial t},\left(\rho_{\varepsilon}^{-1}\right)^{*} \omega\right)_{L_{S}^{2}(0, b)} d t=\int_{0}^{T}\left(A(t) u+\varepsilon A^{\prime}(t) \rho_{\varepsilon}^{-1} u,\left(\rho_{\varepsilon}^{-1}\right)^{*} \omega\right)_{L^{2}(0, b)} d t
$$


The standard integration by parts with respect to $t$ of the left-hand side of (5.5) leads to

$$
\int_{0}^{T}\left(u(\cdot, t), \frac{\partial\left(\rho_{\varepsilon}^{-1}\right)^{*} \omega}{\partial t}\right)_{L_{S}^{2}(0, b)} d t=\int_{0}^{T}\left(A(t) u+\varepsilon A^{\prime}(t) \rho_{\varepsilon}^{-1} u,\left(\rho_{\varepsilon}^{-1}\right)^{*} \omega\right)_{L^{2}(0, b)} d t
$$

The operator $A(t)$ with boundary conditions (2.9) has, on $L^{2}(0, b)$, a continuous inverse. Hence, it is easy to see that

$$
\begin{aligned}
\int_{0}^{T}(u & \left.(\cdot, t), \frac{\partial\left(\rho_{\varepsilon}^{-1}\right)^{*} \omega}{\partial t}\right)_{L_{S}^{2}(0, b)} d t \\
& =\int_{0}^{T}\left(A(t) u+\varepsilon A^{\prime}(t) \rho_{\varepsilon}^{-1} A^{-1}(t) A(t) u,\left(\rho_{\varepsilon}^{-1}\right)^{*} \omega\right)_{L^{2}(0, b)} d t \\
& =\int_{0}^{T}\left(A(t) u+\varepsilon \Lambda_{\varepsilon}(t) A(t) u,\left(\rho_{\varepsilon}^{-1}\right)^{*} \omega\right)_{L^{2}(0, b)} d t \\
& =\int_{0}^{T}\left(A(t) u,\left(I+\varepsilon \Lambda_{\varepsilon}^{*}\right)\left(\rho_{\varepsilon}^{-1}\right)^{*} \omega\right)_{L^{2}(0, b)} d t .
\end{aligned}
$$

The calculations of $A^{-1}(t), \Lambda_{\varepsilon}(t)$, and $\Lambda_{\varepsilon}^{*}(t)$ are straightforward but somewhat tedious. We only give their definitions

$$
\begin{aligned}
A^{-1}(t) g & =\frac{1}{a(t)} \int_{x}^{b} \frac{d \xi}{\xi} \int_{\xi}^{b} g(\eta, t) d \eta+\frac{1}{a(t)} \log \frac{x}{b} \int_{0}^{b} g(x, t) d x, \\
\Lambda_{\varepsilon}(t) g & =a^{\prime}(t) \rho_{\varepsilon}^{-1} \frac{1}{a(\tau)} g(x, \tau), \\
\Lambda_{\varepsilon}^{*}(t)\left(\rho_{\varepsilon}^{-1}\right)^{*} \omega & =\frac{1}{a(t)}\left(\rho_{\varepsilon}^{-1}\right)^{*} a^{\prime}(\tau)\left(\rho_{\varepsilon}^{-1}\right)^{*} \omega .
\end{aligned}
$$

The left-hand side of (5.7) shows that the mapping $\int_{0}^{T}\left(A(t) u, K_{\varepsilon}(t)\left(\rho_{\varepsilon}^{-1}\right)^{*} \omega\right)_{L^{2}(0, b)} d t$ is a continuous linear functional of $u$, where

$$
K_{\varepsilon}(t)\left(\rho_{\varepsilon}^{-1}\right)^{*} \omega=\left(I+\varepsilon \Lambda_{\varepsilon}^{*}(t)\right)\left(\rho_{\varepsilon}^{-1}\right)^{*} \omega,
$$

if the function $K_{\varepsilon}$ has the following properties:

$$
\frac{\partial K_{\varepsilon}}{\partial x} \in L^{2}\left(0, T ; L^{2}(0, b)\right), \quad \frac{\partial}{\partial x}\left(x \frac{\partial K_{\varepsilon}}{\partial x}\right) \in L^{2}\left(0, T ; L^{2}(0, b)\right),
$$

and satisfies

$$
\left.K_{\varepsilon}\right|_{x=0}=\left.K_{\varepsilon}\right|_{x=b}=0 .
$$

Therefore, we conclude from (5.9) and (5.11) that

$$
\left.\left(I+\varepsilon \frac{1}{a(t)}\left(\rho_{\varepsilon}^{-1}\right)^{*} a^{\prime}(\tau)\right)\left(\rho_{\varepsilon}^{-1}\right)^{*} \omega\right|_{x=0}=0 .
$$

For each fixed $x \in[0, b]$ and sufficiently small $\varepsilon$, the operator

$$
\left(I+\varepsilon \frac{1}{a(t)}\left(\rho_{\varepsilon}^{-1}\right)^{*} a^{\prime}(\tau)\right)\left(\rho_{\varepsilon}^{-1}\right)^{*}
$$


has a continuous inverse operator on $L^{2}(0, T)$. Thus from (5.12) we obtain

$$
\left.\omega\right|_{x=0}=\left.\omega\right|_{x=b}=0 .
$$

We set

$$
\omega(x, t)=x^{3} v-3 \mathfrak{I}_{x}^{*}\left(\xi^{2} v\right) .
$$

From (5.14) and (5.15), we conclude that

$$
\int_{0}^{b} x^{2} v(x, t) d x=0, \quad v(b, t)=0 .
$$

If we substitute (5.15) into (5.2), we obtain

$$
\begin{array}{r}
\int_{0}^{T}\left(\frac{\partial u(\cdot, t)}{\partial t}, x^{4} v(\cdot, t)-3 x \mathfrak{J}_{x}^{*}\left(\xi^{2} v(\cdot, t)\right)\right)_{L^{2}(0, b)} d t \\
=\int_{0}^{T}\left(A(t) u, x^{3} v(\cdot, t)-3 \mathfrak{I}_{x}^{*}\left(\xi^{2} v(\cdot, t)\right)\right)_{L^{2}(0, b)} d t
\end{array}
$$

Now we put

$$
u=\mathfrak{I}_{t}\left(e^{c \tau} v\right)=\int_{0}^{t} e^{c \tau} v(x, \tau) d \tau
$$

in (5.17), where $c$ is a constant such that

$$
c c_{0}-c_{2}-36 b^{2} c_{1}^{2} \geq 0
$$

and integrating by parts by taking into account (5.16), we get

$$
\begin{gathered}
\int_{0}^{T}\left(\frac{\partial u(\cdot, t)}{\partial t}, x^{4} v(\cdot, t)-3 x \mathfrak{I}_{x}^{*}\left(\xi^{2} v(\cdot, t)\right)\right)_{L^{2}(0, b)} d t \\
=\int_{0}^{T} \int_{0}^{b} e^{c t} x^{4} v^{2} d x d t+\frac{3}{2} \int_{0}^{T} \int_{0}^{b} e^{c t}\left(\mathfrak{I}_{x}^{*}(\xi v)\right)^{2} d x d t, \\
\int_{0}^{T}\left(A(t) u, x^{3} v(\cdot, t)-3 \mathfrak{I}_{x}^{*}\left(\xi^{2} v(\cdot, t)\right)\right)_{L^{2}(0, b)} d t \\
=-\frac{1}{2} \int_{0}^{b} e^{-c T} a(T) x^{4}\left(\frac{\partial \mathfrak{I}_{T}\left(e^{c t} v\right)}{\partial x}\right)^{2} d x \\
-\frac{1}{2} \int_{0}^{T} \int_{0}^{b} e^{-c t} x^{4}\left(c a(t)-a^{\prime}(t)\right)\left(\frac{\partial \mathfrak{I}_{t}\left(e^{c \tau} v\right)}{\partial x}\right)^{2} d x d t \\
-6 \int_{0}^{T} \int_{0}^{b} x^{3} a(t) v\left(\frac{\partial \mathfrak{I}_{t}\left(e^{c \tau} v\right)}{\partial x}\right) d x d t .
\end{gathered}
$$


Substituting (5.20) into (5.17), yields

$$
\begin{aligned}
\int_{0}^{T} \int_{0}^{b} e^{c t} x^{4} v^{2} d x d t+\frac{3}{2} \int_{0}^{T} \int_{0}^{b} e^{c t}\left(\mathfrak{I}_{x}^{*}(\xi v)\right)^{2} d x d t \\
=-\frac{1}{2} \int_{0}^{b} e^{-c T} a(T) x^{4}\left(\frac{\partial \mathfrak{I}_{T}\left(e^{c t} v\right)}{\partial x}\right)^{2} d x \\
\quad-\frac{1}{2} \int_{0}^{T} \int_{0}^{b} e^{-c t} x^{4}\left(c a(t)-a^{\prime}(t)\right)\left(\frac{\partial \mathfrak{I}_{t}\left(e^{c T} v\right)}{\partial x}\right)^{2} d x d t \\
-6 \int_{0}^{T} \int_{0}^{b} x^{3} a(t) v\left(\frac{\partial \mathfrak{I}_{t}\left(e^{c \tau} v\right)}{\partial x}\right) d x d t .
\end{aligned}
$$

The application of the Cauchy inequality to the last term of the above equality gives

$$
\begin{aligned}
\int_{0}^{T} \int_{0}^{b} e^{c t} x^{4} v^{2} d x d t+\int_{0}^{T} \int_{0}^{b} e^{c t}\left(\mathfrak{I}_{x}^{*}(\xi v)\right)^{2} d x d t \\
\leq-\frac{1}{2} \int_{0}^{b} e^{-c T} a(T) x^{4}\left(\frac{\partial \mathfrak{I}_{T}\left(e^{c t} v\right)}{\partial x}\right)^{2} d x \\
\quad-\int_{0}^{T} \int_{0}^{b} e^{-c t} x^{4}\left(c a(t)-a^{\prime}(t)-36 x^{2} a^{2}(t)\right)\left(\frac{\partial \mathfrak{I}_{t}\left(e^{c T} v\right)}{\partial x}\right)^{2} d x d t
\end{aligned}
$$

According to Assumption 2.1 and inequality (5.19), we get

$$
\begin{aligned}
& \|v\|_{L_{r}^{2}\left(0, T ; L_{\sigma}^{2}(0, b)\right)}^{2}+\|v\|_{L_{r}^{2}\left(0, T ; B_{2, x}^{1, *}(0, b)\right)}^{2} \\
& \quad \leq-\left(c c_{0}-c_{2}-36 b^{2} c_{1}^{2}\right)\|v\|_{B_{2, \rho}^{1}\left(0, T ; L_{\sigma}^{2}(0, b)\right)}^{2} \leq 0
\end{aligned}
$$

and thus $v \equiv 0$, hence $\omega \equiv 0$ almost everywhere in $Q$. This proves Proposition 5.2.

Returning to the proof of Theorem 5.1. Since $F$ is a Hilbert space, the density of $R(L)$ in $F$ is equivalent to the property that orthogonality of a vector $W=\left(\omega, \omega_{0}\right) \in F$ to the range $R(L)$, that is, the identity

$$
\int_{0}^{T}(\mathscr{L} u(\cdot, t), \omega(\cdot, t))_{L_{s}^{2}(0, b)} d t+\left(\ell u, \omega_{0}\right)_{H_{S}^{1}(0, b)}=0, \quad \forall u \in D(L),
$$

implies $W \equiv 0$. In particular, if $u \in D_{0}(L)$ then conclude by Proposition 5.2 that $\omega \equiv 0$. Thus (5.24) implies that

$$
\left(\ell u, \omega_{0}\right)_{H_{S}^{1}(0, b)}=0, \quad u \in D(L) .
$$

Now since $R(\ell)$ is dense in $H_{s}^{1}(0, b)$, it follows that $\omega_{0} \equiv 0$. Hence $\overline{R(L)}=F$. This completes the proof of Theorem 5.1.

ACKNOWLEDGMent. This work was supported by "Le Centre Universitaire Larbi Ben M'hidi de Oum El Bouaghi." 


\section{REFERENCES}

[1] N. I. Benuar and N. I. Yurchuk, A mixed problem with an integral condition for parabolic equations with a Bessel operator, Differentsial nye Uravneniya 27 (1991), no. 12, 2094-2098.

[2] A. Bouziani, Mixed problem with boundary integral conditions for a certain parabolic equation, J. Appl. Math. Stochastic Anal. 9 (1996), no. 3, 323-330.

[3] _ Solution forte d'un problème mixte avec condition intégrale pour une classe d'équations paraboliques, Maghreb Math. Rev. 6 (1997), no. 1, 1-17 (French).

[4] _ On a third order parabolic equation with a nonlocal boundary condition, J. Appl. Math. Stochastic Anal. 13 (2000), no. 2, 181-195.

[5] __ On a class of nonclassical hyperbolic equations with nonlocal conditions, to appear in J. Appl. Math. Stochastic Anal., 2002.

[6] - On the quasi-static flexure of a thermoelastic rod, to appear in Commun. Appl. Anal., 2002.

[7] A. Bouziani and N. E. Benouar, Problème mixte avec conditions intégrales pour une classe d'équations paraboliques, C. R. Acad. Sci. Paris Sér. I Math. 321 (1995), no. 9, 11771182 (French).

[8]___ Mixed problem with integral conditions for a third order parabolic equation, Kobe J. Math. 15 (1998), no. 1, 47-58.

[9] S. Mesloub and A. Bouziani, Problème mixte avec conditions aux limites intégrales pour une classe d'équations paraboliques bidimensionnelles, Acad. Roy. Belg. Bull. Cl. Sci. (6) 9 (1998), no. 1-6, 61-72.

[10] _ On a class of singular hyperbolic equation with a weighted integral condition, Int. J. Math. Math. Sci. 22 (1999), no. 3, 511-519.

[11] N. I. Yurchuk, Solvability of boundary value problems for certain operator-differential equations, Differensial'nye Uravneniya 13 (1977), no. 4, 626-636.

[12] __ A mixed problem with an integral condition for some parabolic equations, Differentsial nye Uravneniya 22 (1986), no. 12, 2117-2126.

AbDelfatah Bouziani: DÉpartement de MATHÉmatiques, Centre UniVERsitaire LaRbi BeN M'Hidi-OUM El BOUAGUi, B.P. 565, 04000, AlgeRIA

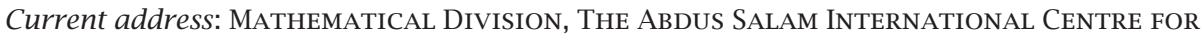

Theoretical Physics (ICTP), Strada Costiera 11, 34100 Trieste, ITALY

E-mail address: bouziani@ictp.trieste. it 


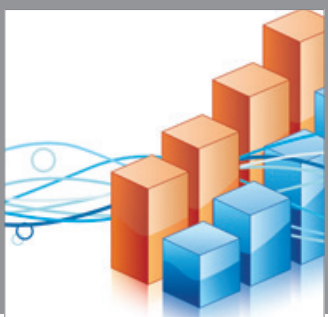

Advances in

Operations Research

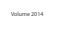

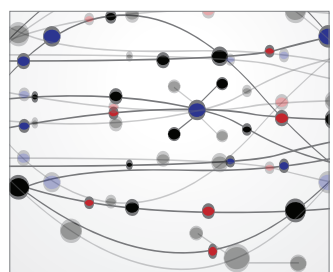

\section{The Scientific} World Journal
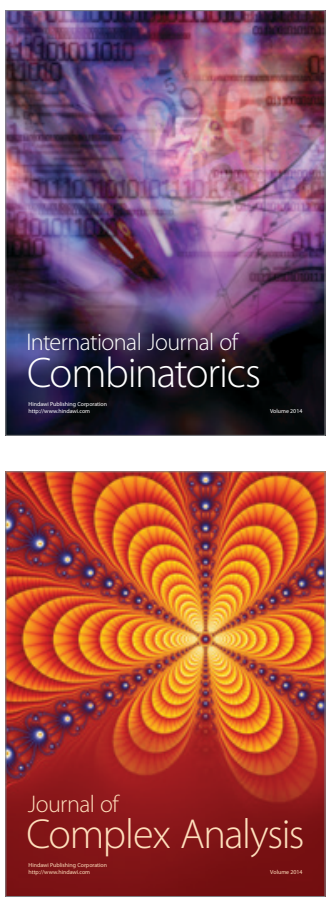

International Journal of

Mathematics and

Mathematical

Sciences
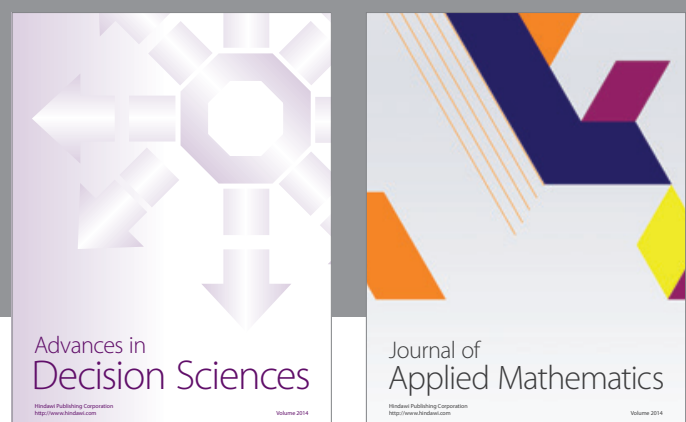

Journal of

Applied Mathematics
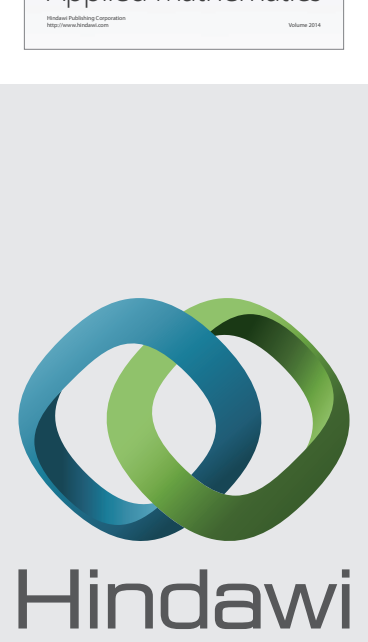

Submit your manuscripts at http://www.hindawi.com
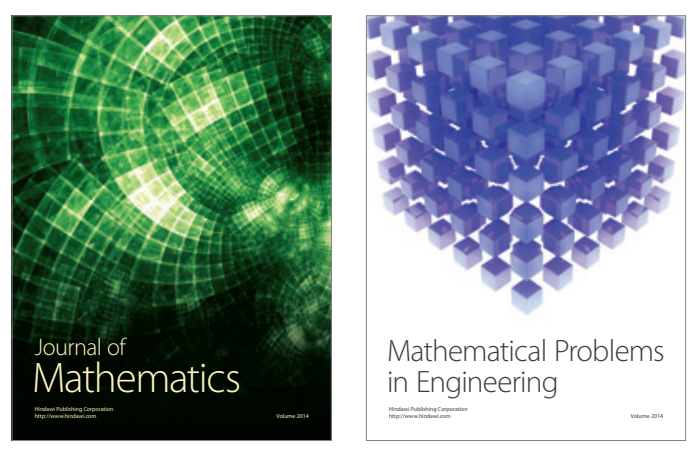

Mathematical Problems in Engineering
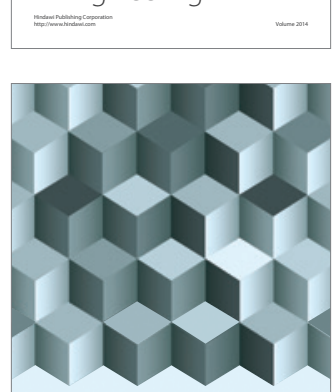

Journal of

Function Spaces
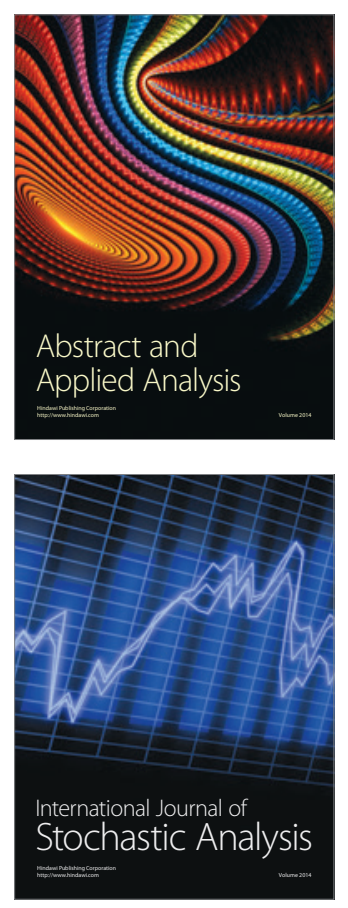

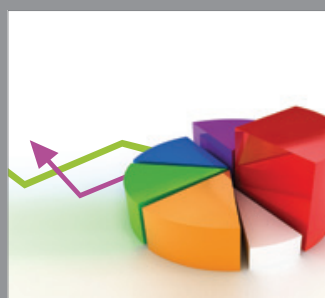

ournal of

Probability and Statistics

Promensencen
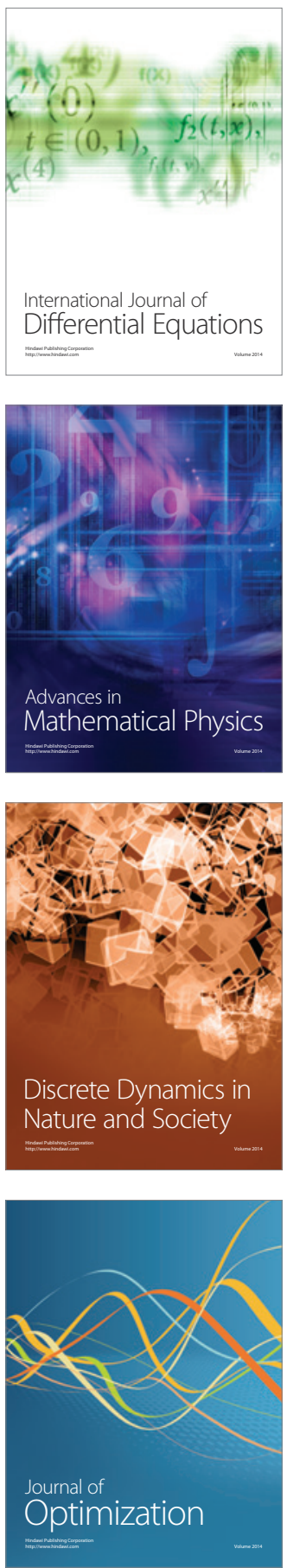